\title{
Relationships between Leaf and Stem Soluble Sugar Content and Tuberous Root Starch Accumulation in Cassava
}

\author{
Xinglu Luo \& Qiufeng Huang \\ Agricultural College, Guanxi University, Nanning 530005, Guanxi, China \\ E-mail: luoxinglu@sina.com
}

Received: September 9, $2010 \quad$ Accepted: September 26, 2010 doi:10.5539/jas.v3n2p64

\begin{abstract}
The purpose of this study was to examine the relationships between the soluble sugar content in the leaves and stems and the starch accumulation in the tuberous roots of four cassava cultivars: FuXuan01, GR891, SC124, and SC201. The results showed that the highest leaf soluble sugar content was in September, and the highest stem sugar content was in October, which played an important role in starch accumulation in the tuberous roots. The leaf and stem soluble sugar content and the tuberous root starch accumulation were positively correlated in the setting and growth stages but negatively correlated in the ripening stage. The soluble sugar content in the leaves went from high to low as the tuberous roots progressed from the setting stage to the ripening stage; starch accumulation in the root tubers followed the opposite pattern.
\end{abstract}

Keywords: Cassava, Leaves, Soluble sugar contents, Starch accumulation, Stems

\section{Introduction}

Cassava is a woody shrub that is extensively cultivated as an annual starch crop for its edible, tuberous root. One of the primary goals in cassava cultivation and breeding has been to obtain rich starch content in the root (Xing-lu 2001, Songhai 1994, Qiu-ying2000). Cassava has a long growth period, and cassava yielding and starch accumulation occur with the late stage of breeding. Studying the physiological mechanism of cassava starch accumulation in its late breeding stage and utilizing the most appropriate methods to increase starch accumulation is an effective way to obtain high starch content in the tapioca root. Shu-li et al. (2005) previously reported that in potato, rice, barley, wheat, corn, and sweet potato, starch accumulation relies largely on the synthesis, translocation and accumulation of the internal soluble sugar (Shu-li et al 2005). Further, it has been demonstrated that the soluble sugar in leaves also had an effect on the starch accumulation in the tuberous root (Xing-lu2006). Previous research has shown that the change of the soluble sugar content in wheat leaves and stems was correlated with the starch accumulation in the seeds and the trend was that increasing of soluble sugar content in leaves and stems in early stage and decreasing in late stage was good for starch accumulation in the seeds. (Jiang Dong2001, You-jun 2005, Jian-min2008, Bao-ren2007, Jin-zhi2008, Xiao-jie2008, Chun-yan2007, Hai-ying,2006, Xue-li2005, Chen-yang2005, WANG Jue2008, Wen-jing2007, Zhong-min2007, Xu-dong2003, etc). It has been reported that soluble sugar content in rice leaves is closely related to starch accumulation in the grain (ZHANG Jun2008, WANG Wei2006, Yan-fang2006), yet such relationships within the cassava plant have not been investigated, either in China or elsewhere. Therefore, the present study investigated the relationship between the change in soluble sugar in cassava leaves, stems and tuberous roots and the starch accumulation in tuberous roots under open field cultivation conditions, which provides a theoretical basis for physiological control in the breeding and the cultivation of high starch content and high yield cassava cultivars.

\section{Materials and Methods}

\subsection{Trial cassava cultivars}

The cassava cultivars for trial included two high-starch cassava cultivars (Fuxuan 01 and GR891) and two low-starch cultivars (SC124 and SC201).

\subsection{Test design}

The experiment was conducted in a mid-soil fertility sample plot at the teaching and scientific research base of the Agricultural College of Guangxi University. A randomized block design was utilized. Each block was repeated three times, and the area of each block was $42 \mathrm{~m}^{2}$ with a planting specification of $80 \times 80 \mathrm{~cm}$. The 
cultivars were planted in April 2007 and harvested in mid-January 2008, and the cultivation management was the same as that of normal open field cultivation.

\subsection{Measured parameters and methods}

\subsubsection{Determining reducing sugar content}

Reducing sugar content was measured using a method with 3,5-dinitrosalicylic acid (DNS) described in Analysis Techniques for Grain Quality of Cereals and Oils by Zhaofan (1985). Briefly, a 0.3-0.5 g dried sample (shattered, fineness: passing through 40-mesh) was weighed in a triangular flask. Twenty milliliters of distilled water was added, and the sample was heated in a $90-99^{\circ} \mathrm{C}$ water bath for $20 \mathrm{~min}$, then cooled down to room temperature. The extracted solution was transferred to a flask and mixed with distilled water to a total volume of $50 \mathrm{~mL}$. The solution was filtered, and $1 \mathrm{~mL}$ of DNS reagent per $1 \mathrm{~mL}$ of the filtered solution was added. The mixture was heated in a boiling water bath for $5 \mathrm{~min}$ and then cooled down with tap water. Eight milliliters of distilled water was added, and the solution was mixed well. The absorbency at $520 \mathrm{~nm}$ wavelength was measured.

Content of reducing sugar $(\%)=\left[(\mathrm{A} \times 50) /\left(1 \times \mathrm{W} \times 10^{3}\right)\right] \times 100=5 \mathrm{~A} / \mathrm{W}$

Where: $\mathrm{A}-$ reducing sugar content in the sample solution to be measured obtained by referring to the standard curve (mg)

$$
\begin{aligned}
& \mathrm{W} \text {-weight of the sample }(\mathrm{g}) \\
& 50 \text { - total volume of the sample solution }(\mathrm{mL}) \\
& 1 \text { - volume of the sample solution to be measured }(\mathrm{mL}) \\
& 10^{3} \text { - milligram converted from gram } \\
& 100 \text { - converted percentage composition }
\end{aligned}
$$

\subsubsection{Determining sucrose content}

To measure sucrose content, the anthrone colorimetric method described in the Experimental Handbook of Plant Physiology by Shanghai Society for Plant Physiology (1985) was applied. Briefly, $0.1 \mathrm{~g}$ of dried sample (shattered, fineness: passing through 100 -mesh) was weighed in a $10 \mathrm{~mL}$ centrifuge tube, and $6-7 \mathrm{~mL}$ of $80 \%$ ethanol was added. The sample was heated in an $80^{\circ} \mathrm{C}$ water bath for $30 \mathrm{~min}$ and centrifuged $(3000 \mathrm{r} / \mathrm{min})$ for 5 $\mathrm{min}$. The supernatant was collected and the extraction was repeated twice $(3000 \mathrm{r} / \mathrm{min}$ for $10 \mathrm{~min}$ each). Supernatant was collected into a flask and $80 \%$ ethanol was added to a final volume of $50 \mathrm{~mL}$. Then $1 \mathrm{~mL}$ of the extracted solution was added to a test tube and heated in a boiling water bath until it condensed to $0.05-0.1 \mathrm{~mL}$ (not greater than $0.1 \mathrm{~mL}$ to avoid longer color developing time). Next, $0.1 \mathrm{~mL}$ of $30 \% \mathrm{KOH}$ was added and the solution was incubated in boiling water for $10 \mathrm{~min}$. After the solution cooled down to room temperature, $3 \mathrm{~mL}$ of anthrone reagent was added, and the solution was incubated at $40^{\circ} \mathrm{C}$ for $10-15 \mathrm{~min}$. The absorbency at $620 \mathrm{~nm}$ wavelength was measured.

Sucrose content $(\%)=\left\{[\mathrm{C} \times(\mathrm{V} / \mathrm{a})] /\left(\mathrm{W} \times 10^{6}\right)\right\} \times 100$

where: $\mathrm{C}$-glucose content obtained by referring to the standard curve $(\mu \mathrm{g})$

$\mathrm{V}$ - total volume of the extracted solution $(\mathrm{mL})$

$\mathrm{a}-$ volume of sample solution for color developing $(\mathrm{mL})$

$\mathrm{W}$-weight of sample (g)

\subsubsection{Total soluble sugar content measurement}

To measure total soluble sugar content, the anthrone colorimetric method described in Research Method in Crop Physiology by Xianzheng (1992) was applied. One-tenth of a gram dried sample (shattered, fineness: passing through 100-mesh) was weighed in a $10 \mathrm{~mL}$ centrifuge tube, to which $6-7 \mathrm{~mL}$ of $80 \%$ ethanol was added. The sample was heated in an $80^{\circ} \mathrm{C}$ water bath for $30 \mathrm{~min}$, then centrifuged $(3000 \mathrm{r} / \mathrm{min})$ for $5 \mathrm{~min}$. The supernatant was collected, and the extraction was repeated twice (3000 $\mathrm{r} / \mathrm{min}$ for $10 \mathrm{mins}$ each). The supernatant was collected into a flask, and $80 \%$ ethanol was added to total volume of $50 \mathrm{~mL}$. Then, $1 \mathrm{~mL}$ of solution was taken, and $1.5 \mathrm{~mL}$ of water was added, followed by $6.5 \mathrm{~mL}$ of anthrone reagent. The sample was mixed and incubated at room temperature $\left(18-30^{\circ} \mathrm{C}\right)$ for $15 \mathrm{~min}$ to allow color developing. The absorbency at $620 \mathrm{~nm}$ wavelength was measured after the sample was cooled down.

Content of total soluble sugar $(\%)=\left\{[\mathrm{C} \times(\mathrm{V} / \mathrm{a})] /\left(\mathrm{W} \times 10^{6}\right)\right\} \times 100$

Where: $\mathrm{C}$ - glucose content obtained by referring to the standard curve $(\mu \mathrm{g})$ 


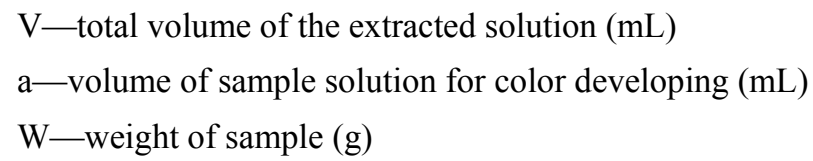

\subsubsection{Determining starch content in tuberous roots}

The residual sugar content measurement was rinsed with $20-30 \mathrm{~mL}$ distilled water in a $50 \mathrm{~mL}$ flask. The solution was gelatinized in boiling water for $15 \mathrm{~min}$ and cooled down. Two milliliters of cold $9.2 \mathrm{~mol} / \mathrm{L}$ perchloric acid was added, and the mixture was gelatinized again in boiling water for $15 \mathrm{~min}$. Distilled water was added to a final volume of $50 \mathrm{~mL}$, and the solution was mixed. Five $\mathrm{mL}$ of anthrone reagent was added to $2 \mathrm{~mL}$ of the supernatant. The solution was boiled for $10 \mathrm{~min}$. The absorbency at $620 \mathrm{~nm}$ wavelength was measured after cooling.

Starch content $(\%)=\{[\mathrm{C} \times(\mathrm{V} / \mathrm{a}) \times 0.9] /(\mathrm{W} \times 106)\} \times 100$ where: 0.9 -the starch coefficient from glucose conversion;

C- glucose value $(\mu \mathrm{g})$ obtained by referring to the standard curve;

$\mathrm{V}$ - total volume of the extracted solution $(\mathrm{mL})$

$\mathrm{a}$ - volume of sample solution for color developing $(\mathrm{mL})$

$\mathrm{W}$-weight of sample (g)

\section{Results and Analysis}

\subsection{Changes in total soluble sugar content in different parts of various cassava cultivars}

Soluble sugars, including monosaccharides and oligosaccharides, are the main photosynthetic product and the main substrate for metabolism (Oscar G.,2010) Carbohydrate content accounts for $90-95 \%$ of the dry matter in a plant. Soluble sugar is the major form of carbohydrate that can be reciprocally transformed and reused by plants. The high content of soluble sugar in cassava leaves facilitates the translocation of photosynthetic products from the stem to the tuberous root as well as the nutrient accumulation in the root. This is one of the reasons for the decreased content of total soluble sugar in the reproductive growth period of cassava.

There were no major differences in the development trend of soluble sugar in the leaves of different cultivars (Fig.1). The soluble sugar content in cassava leaves increased rapidly from August to September, then dropped. The content of soluble sugar after October was much lower in Fuxuan 01 than in other cultivars.

The change in soluble sugar content in cassava stems developed in a single-peak curve (Fig. 2). The soluble sugar content of Fuxuan 01, GR891 and SC124 reached their peaks in October while that of SC201 was highest in November and then dropped afterwards. The soluble sugar content of the high-starch cultivar GR891 was much lower than that of other cultivars.

Additionally, the change in the content of soluble sugar of tuberous roots had two wave peaks during the measuring period, one of which occurred in September and the other in November (Fig.3). The soluble starch content in high-starch cultivars was significantly lower than those of the low-starch cultivars, indicating that the high-starch cultivars had better photosynthesis ability than the low-starch cultivars and ensuring the forming of starch in the tuberous roots.

\subsection{Changes in sucrose content in different parts of different cassava cultivars}

Sucrose is the major form of saccharide for long distance transportation in the sieve tubes of higher plants. By comparing the soluble sugar and sucrose contents in the cassava leaves and stems, it is apparent that the changes of sucrose contents and soluble sugar contents in the leaves and stems of different cassava cultivars maintained similar trend lines (Fig. 4 and Fig. 5). Specifically, the sucrose contents in the tuberous roots of various cassava cultivars was decreased in August and September and rapidly bounced back until reaching the peak in October-November followed by a quick drop (Fig. 6). During the recovery, the sucrose content in low-starch cultivars was significantly higher than that of the high-starch cultivars, indicating a quicker starch accumulation rate in the latter. This resulted in a continuous transformation of reducing sugar from sucrose.

\subsection{Changes in reducing sugar contents in different parts of different cassava cultivars}

All monosaccharides and most disaccharides and oligosaccharides have reducing properties and can become reducing sugars. Reducing sugar is the major soluble sugar in leaves, accounting for over $74 \%$ of the total sugar. Cassava leaves contained the richest reducing sugar content followed by the stems and the tuberous roots, and the reducing sugar content in the tuberous roots of high-starch cultivars was lower than those of the low-starch 
cultivars. The contents of the reducing sugar in the stems and tuberous roots of different cultivars were decreased during the measured period (Fig. 7-9). This may be due to the enhanced ability to utilize and transform the reducing sugar, along with the increased starch content in the tuberous roots throughout the growth period.

\subsection{Changes in starch contents and accumulation rates in tuberous roots of different cassava cultivars}

The root is the main harvest product of cassava, and its essential component is starch. The starch content of different cassava cultivars varies depending on growth periods. This is an indicator of the differences in their individual photosynthetic rates, as well as the ability of carrying, transporting and unloading starch in the tissue pools. Further, it also indicates that there are differences in the ability to transform sugar into starch in the tuberous roots. The starch content of different cassava cultivars increased gradually, among which GR891 contained the richest starch (Table 1). Analysis results showed that the differences in starch contents in tuberous roots between high-starch and low-starch cassava cultivars during this measured period reached significant levels.

\section{Discussion}

Previous findings showed that the starch accumulation in the tuberous roots had a positive correlation with the soluble sugar content in cassava leaves and a negative correlation with the reducing sugar content in leaves as well as the sucrose contents in leaves (LUO Xing-lu, 2002). It has also been shown that high-yield crops required not only strong carbon assimilation in leaves but also effective photosynthetic product transportation and distribution from leaves to the pool end. Cassava is a $\mathrm{C}_{3}-\mathrm{C}_{4}$ intermediate plant with very high photosynthetic efficiency(EL-Sharkawy et al,1990). Photosynthesis produces sucrose in the leaves. The soluble sugar content in the leaves of both high and low starch cassava cultivars is the highest in the whole plant, indicating that the source leaves are of very strong capacity for timely transport of nutrients, which facilitates the transport of sugar to the tuberous root pools so as to provide sufficient substrate supply for starch synthesis.

Jiang Dong et al. (2001) and Youjun et al. (2005) reported studies on the changes in soluble sugar contents in wheat canes, Yet no studies on the relationship between the change in soluble sugar in cassava cane and starch accumulation in the tuberous root have been reported. The present study was conducted to investigate the soluble sugar content in the stems of different cassava cultivars. Results showed the contents of soluble sugar in stems of different cassava cultivars presented single-peak curves characterized by a rising trend in the early stage. The peaks of soluble sugar contents of the sampled Fuxuan 01, SC124 and SC121occured in September while that of the high-starch GR891 cultivar occurred in October, which was the lowest content among all cultivars. This indicates that there is a difference in the soluble sugar contents in different tissues and cassava cultivars.

Soluble sugar is the substrate for starch synthesis and there is a close relationship between the soluble sugar content and starch synthesis in plants. Previous studies have demonstrated this phenomenon. It has long been maintained that there was a relationship between starch synthesis and soluble sugar content in plants, however, such a relationship is complicated and not well understood SHEN Songhai et al,1994; Binbin Lu et al,2005). Results hereby show that, in general, the high-starch cassava cultivars Fuxuan 01 and GR891 contained lower soluble sugar in tuberous roots than that in the low-starch cassava cultivars SC124 and SC201, indicating that the former cultivars had a better pool-source relationship,. Therefore, the high-starch cultivars had more starch transformation from soluble sugar, which leads to lower content of soluble sugar in the tuberous roots.

Reducing sugar and sucrose are the two main soluble sugars in cassava, of which the former is the immediate product of photosynthesis as well as the prerequisite substance for sucrose synthesis, while and the latter is the major form of organics in internal transport. During the first stage of the later growth period (October 19 November 19), as the cassava cultivars continued to grow, the sucrose content in leaves increased while the reducing sugar content decreased; they both dropped in the stems. During the second stage (November 19 December 19), the sucrose content in the leaves dropped while the reducing sugar content relatively increased; the sucrose content in stems decreased while the reducing sugar content increased except for that of the high-starch cultivar Fuxuan 01. This pattern suggests that leaves transform reducing sugar into sucrose rapidly during the first stage and transport it to the stems and roots for starch synthesis, leading to the decrease of sucrose in leaves during the second stage. Due to weak starch synthesis ability in the tuberous roots, the sucrose to be transported to the roots also decreased during the second stage (harvest). As a result, the rate of transformation from reducing sugar into sucrose in the leaves decreased, which explains the relative increase reducing sugar in leaves during the later stage. Therefore, maintaining a continual sucrose supply in the tuberous roots is a prerequisite for high starch accumulation in the roots. In conclusion, enhancing the photosynthetic ability in leaves, as well as the distribution and storage ability of photosynthesis products in the nutritive organ may be a most effective way to increase starch yield in the roots of cassava. 


\section{References}

Binbin Lu et al. (2005). Modulation of keyenzymes involved inammonium assimilation and Carbon metabolism by low temperature in rice (Oryzasativa L.)roots. Plant Science, (169), 295-300.

DAI Zhong-Min et al. (2007). Characterization of Starch Accumulation and Activities of Enzymes Involved in Starch Synthesis in Grains of Wheat Cultivars Differing in Spike Types Field-Grown in Irrigation and Rainfed Conditions. Act Agron Sin, 33(4):164-167.

EL-Sharkawy et al. (1990). Relationship between biomass, root-yield and single-leaf photosynthesis in field-grown cassava. Field Crop Res., (34):183-201.

FU Xue-li et al. (2005). Effects of Interaction of Irrigation Times and Nitrogen Application Rates on Sucrose Content and Starch Accumulation in Both Flag Leaves and Grains of Weak-Gluten Wheat. J Triticeae Crops, 25(4):73-77.

He Zhaofan. (1985). Analysis Techniques for Grain Quality of Cereals and Oils. Bejing. Chinese Agric, Press, 151-152.

JIANG Dong et al. (2001). Changes of Soluble Sugar Contents in Leaf, Stem and Grain in Winter Wheat and Its Relationship with Grain Starch Accumulation. J Triticeae Crops, 21(3): 38-41.

LI Chun-yan et al. (2007). Relationship of Grain Starch Synthesis with Changes of Endogenous Hormone and Sucrose Content after Anthesis in Wheat Variety Ningmai 9. J Triticeae Crops, 27(1): 146-150

LI You-jun et al. (2005). Studies on the Dynamic Changes of Soluble Sugar Contents in Leaf, Stem and Grain in Different Winter Wheats and the Relationship with Grain Starch Contents. Sci Agri Sin, 38(11): 2219-2226

LI Jian-Min et al. (2008). Activities of Enzymes Involved in Sucrose and Starch Synthesis during Grain Filling and the Relation to Nitrogen Metabolism in Strong-and Weak-Gluten Wheat Cultivars. Acta Agron Sin, 34(6): 952-957.

LIU Hai-ying et al. (2006). Effect of Epibrassinolide Sprayed at Anthesis on Sucrose Metabolism and Yield of Yumai 49. Journal of Triticeae Crops, 26(1): 85-89.

LUO Xing-lu, Lao Tian-Yuan. (2001). Studies on The characters of Growth and Starch Accumulation of Several Cassava Varieties. Chin Agric Sci Bull, 17(4): 22-26.

LUO Xing-lu et al. (2006). Studies on The Relationship between Soluble Sugar Content in The Leaves And The Starch accumulation in The Root Tuber of Cassava. Chin Agric Sci Bulletin, 8:289-291.

Oscar G. Bodeln, MaraBlanch, Mara T. Sanchez-Ballesta, Mara I. Escribano, Carmen Mer. (2010). The effects of high $\mathrm{CO}_{2}$ levels on anthocyanin composition, antioxidant activity and soluble sugar content of strawberries stored at low non-freezing temperature. Food Chemistry, (122), 673-678.

SHEN Songhai et al. (1994). Relation of starch accumulation with free sugars in the sweet potato (Lpomoea batatas). J Agric Univ Zhejiang, 20(4):400-404.

Shanghai Society of Plant Physiologists. (1985). The Experimental Handbook of Plant Physiology. Shanghai. Shanghai Sci and tech Press, 137-150.

WANG Chen-yang et al. (2005). Advances in Starch Synthesis, Starch Properties in Wheat Grain and Their Agronomic Regulation. J Triticeae Crops, 25(1): 119-124.

WANG Jue et al. (2008). Effects of High Temperature after Anthesis on Starch Traits of Grain in Wheat. $J$ Triticeae Crops, 28(2):89-94.

WANG Shu-li et al. (2005). Soluble Sugar Contents in Leaf and Grain in Two Gluten Wheats and Its Relationship with Grain Starch Accumulation. J HENAN Agric Sci, (4):12-15.

WU Jin-zhi et al. (2008). Effects of Different Nitrogen Forms on Grain Starch Accumulation and the Activities of Related Enzymes in Weak Gluten Wheat. J Triticeae Crops, 28(1): 124-129.

WANG Wen-Jing et al. (2007). Dynamic Changes of Activities of Key Enzymes Involved in Sucrose Metabolism during Grain Filling in Wheat and the Relationship with Starch Accumulation in Grain. Acta Agron Sin, 33(7): 82-88.

WANG Xu et al. (2003). Effect of Potassium on Sucrose Content of Flag Leaves and Starch Accumulation of Kernels in Wheat. Acta Phytoecologica Sin, (2):57-62.

WANG Xu-dong et al. (2003). Effect of potassium on carbohydrate contents in stem and sheathand starch 
accumulation in kernel of wheat. Plant Nutr and Fert Sci, (1):56-61.

WANG Wei et al. (2006). Effect of Controlled Soil Drying on Grain Filling and Activities of Key Enzymes for Starch Synthesis in Rice. Acta Agron Sin, 32(7):28-35.

WANG Yan-fang et al. (2006). Relationship Between Soluble Sugar Contents and Starch Accumulation in Grain During Grain-filling Stage in Spring Maize. J Maize Sci, (2):87-89.

Zhang Xian-zheng. (2000). Research Method in Crop Physiology, Bejing: High Educat Press, 193-197.

ZHANG Qiu-ying et al. (2000). Relationship between Starch, Protein Accumulation and Substrate Supply during Grain Filling in Spring Wheat. $J$ Triticeae Crops, 20(1):55-58.

ZHANG Bao-ren et al. DONG Shu-tin HU Chang-hao WANG Kong-juon. (2007). Effect of High Air Temperature during Different Growth Stage on Starch Synthesis in Grain and Yield in Maize (Zea mays L.) Acta Agron Sin, 33(1):32 -39.

ZHU Xiao-jie et al. (2008). Effects of Sulfur Application on Sugar Content of Flag Leaves and Starch Accumulation of Kernels in Wheat. Journal of Triticeae Crops, 28(3): 121-125.

ZHANG Jun et al. (2008). Effects of Nitrogen on Starch Accumulation and Activities of Enzymes Involved in Starch Synthesis in Rice Caryopsis. Acta Agron Sin, 34(12): 114-12.

ZHANG Xian-zheng. (1992). Resaerch Methods of Crop Physiology. Bejing: Chin Agric Press, 148-150.

Table 1. The starch accumulating in the tuberous roots of different cassava cultivars (\%)

\begin{tabular}{lcccccc}
\hline Cultivar & June19th & July19th & Aug. 19th & Setp. 19th & 0ct. 19 th & Harvest period \\
\hline FX01 & 12.9 & 14.5 & 15.8 & 20.8 & 27.9 & 31.9 \\
GR891 & 13.5 & 14.9 & 16.5 & 21.3 & 28.4 & 33.8 \\
SC124 & 12.6 & 13.9 & 15.4 & 18.4 & 23.9 & 26.2 \\
SC201 & 12.3 & 13.2 & 15.1 & 18.3 & 23.2 & 25.8 \\
\hline
\end{tabular}

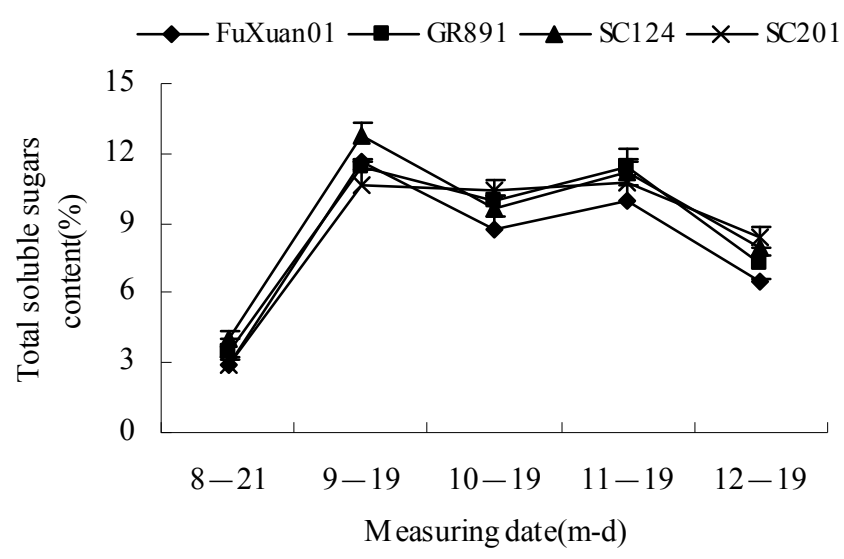

Figure 1. The changes of soluble sugar in the leaves of different cassava cultivars 


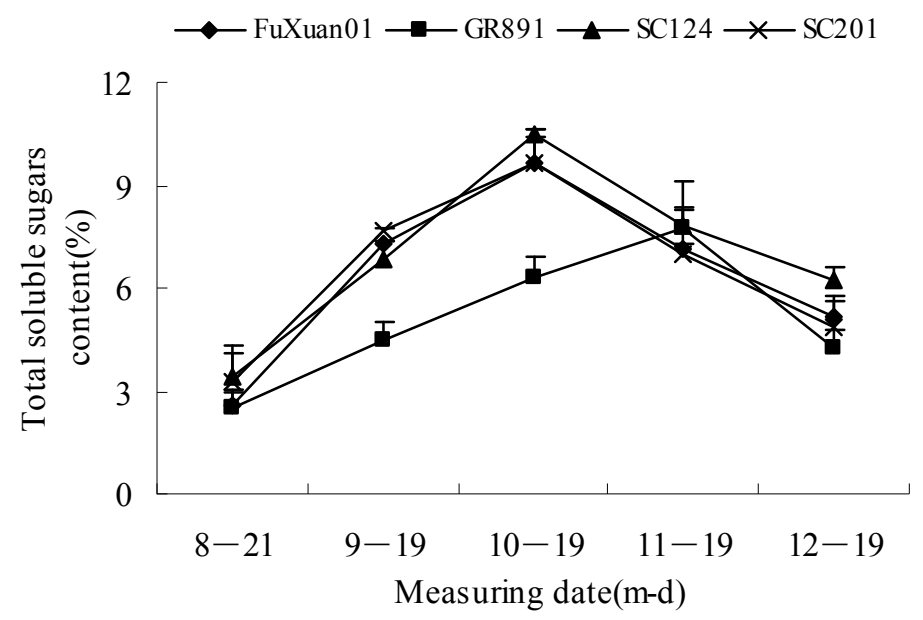

Figure 2. The change of soluble sugar contents in cassava stems of different cassava cultivars

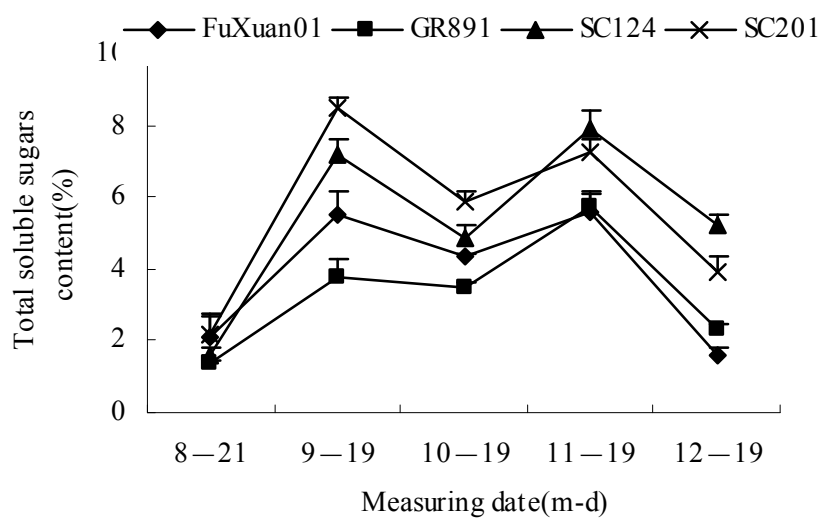

Figure 3. The change of the content of soluble sugar of tuberous roots of different cassava cultivars

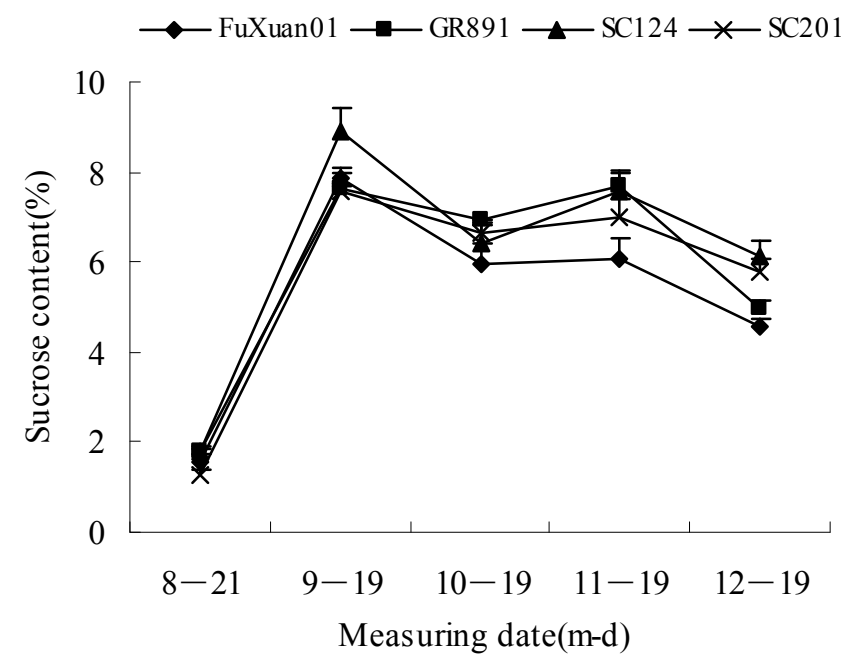

Figure 4. The changes of sucrose contents in the leaves of different cassava cultivars 


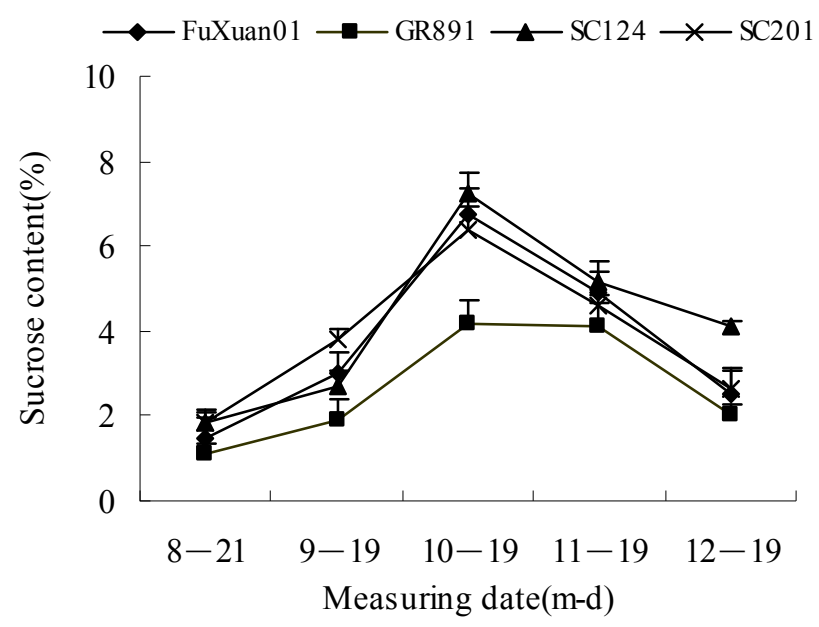

Figure 5. The changes of sucrose contents in the stems of different cassava cultivars

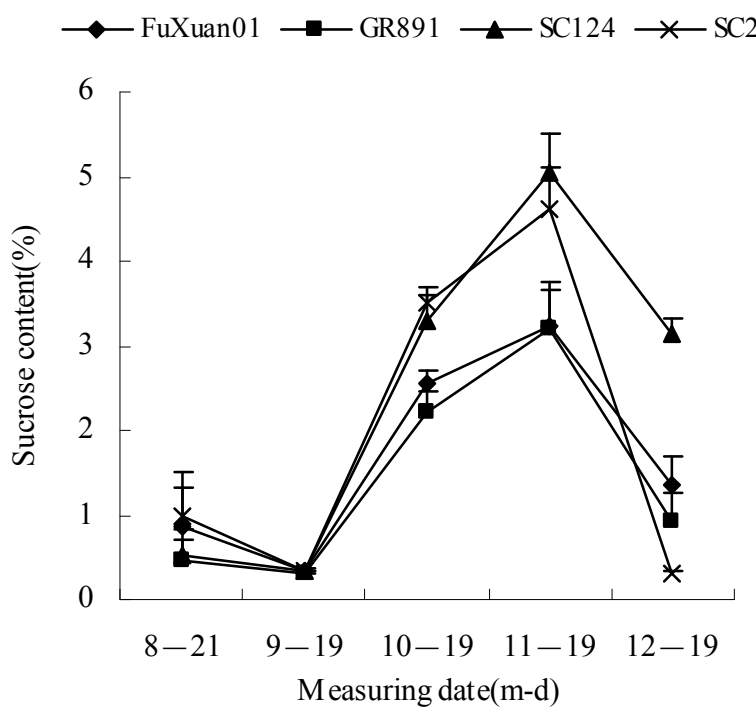

Figure 6. The changes of sucrose contents in the tuberous roots of different cassava cultivars

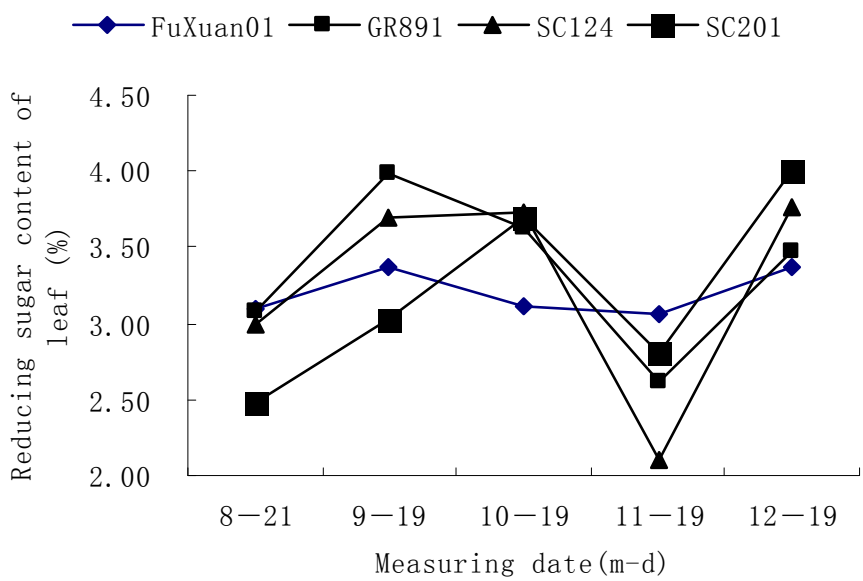

Figure 7 . The changes of reducing sugar content in the leaves of different cassava cultivars 


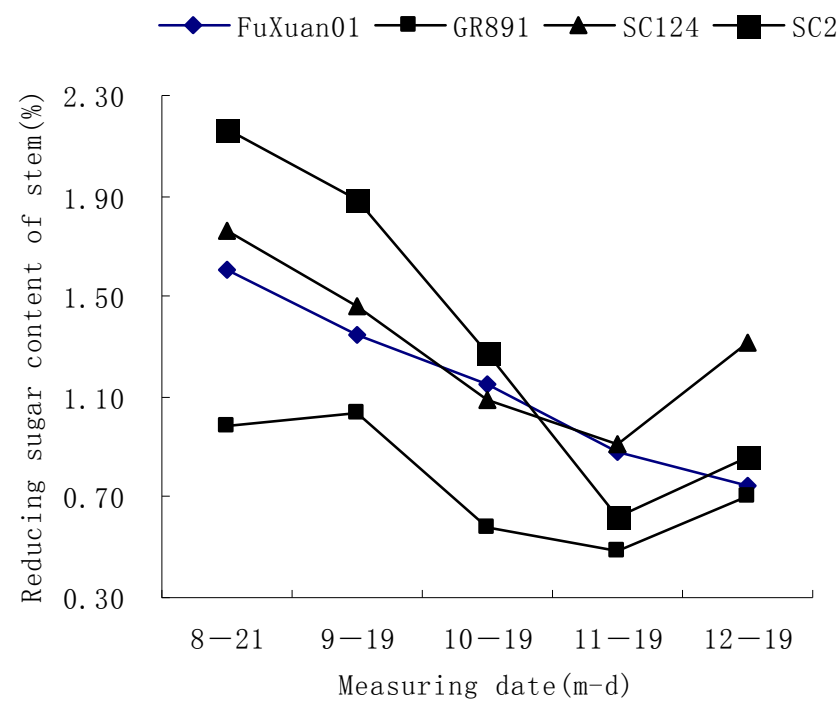

Figure 8 . The changes of reducing sugar content in the stems of different cassava cultivars

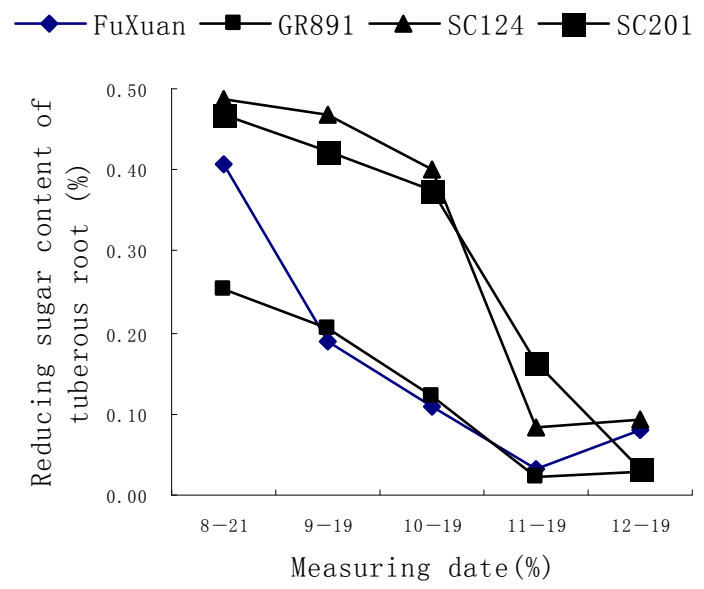

Figure 9. The changes of reducing sugar content in the tuberous roots of different cassava cultivars 